\title{
NAPNE: ANÁLISE DAS RESOLUÇÕES DOS INSTITUTOS FEDERAIS DE DIFERENTES REGIÕES
}

\author{
NAPNE: ANALYSIS OF THE RESOLUTIONS OF FEDERAL INSTITUTES OF
}

\author{
DIFFERENT REGIONS
}

\author{
Jussara Cristina Rodrigues da Silva ${ }^{1}$ \\ Michele Oliveira da Silva²
}

\begin{abstract}
RESUMO: O presente estudo objetivou analisar as Resoluções que regulamentam a criação dos NAPNEs - Núcleos de Apoio às Pessoas com Necessidades Educacionais Específicas em um Instituto Federal de Educação, Ciência e Tecnologia por região do Brasil: Norte, Nordeste, Centro-Oeste, Sudeste e Sul. O NAPNE é um núcleo responsável por favorecer a inclusão nessas Instituições de ensino que têm apresentado diferenças entre as suas Resoluções de criação. Para o estudo proposto, foi realizada uma pesquisa exploratória, com abordagem qualitativa. O método escolhido para o desenvolvimento do estudo foi a pesquisa documental. A análise possibilitou a identificação da predominância das diferenças nas Resoluções quanto à sua nomenclatura e sigla, objetivo/finalidade, público-alvo, competências, composição e financiamento. O resultado dessa pesquisa contribuirá com a reflexão sobre os limites e possibilidades apresentadas nas próprias Resoluções, evidenciando-se, assim, a necessidade da elaboração de uma diretriz nacional que minimize as disparidades encontradas entre os NAPNEs.
\end{abstract}

Palavras-chave: NAPNE. Diferenças. Semelhanças. Resoluções. Institutos Federais.

ABSTRACT: This study aimed to analyze the Resolutions that regulate the creation of NAPNEs - Centers for Support of People with Specific Educational Needs in a Federal Institute of Education, Science and Technology by region of Brazil: North, Northeast, Midwest, Southeast and South. NAPNE is a nucleus responsible for promoting inclusion in these educational institutions that have presented differences between their resolutions of creation. For the proposed study, an exploratory research was carried out, with a qualitative approach. The method chosen for the development of the study was documentary research. The analysis allowed the identification of the predominance of differences in resolutions regarding their nomenclature and acronym, objective/purpose, target audience, competencies, composition and financing. The result of this research will contribute to the reflection on the limits and possibilities presented in the Resolutions themselves, thus evidencing the need to develop a national guideline that minimizes the disparities found between NAPNEs.

Keywords: NAPNE. Differences. Similarities. Resolutions. Federal Institutes.

1Assistente Social e Representante do NAPNE do Instituto Federal de Educação, Ciência e Tecnologia de São Paulo - Campus Itapetininga. E-mail: jucoloro@gmail.com. Mestranda em Estado, Gobierno y Políticas Públicas pela Flacso Brasil.

2Pedagoga e Representante do NAPNE do Instituto Federal de Educação, Ciência e Tecnologia de São Paulo Campus Birigui. E-mail: micheleoliveira@ifsp.edu.br. Doutora em Educação pela UNESP. 


\section{INTRODUÇÃO}

A lei de criação dos Institutos Federais de Educação, Ciência e Tecnologia foi sancionada em 29 de dezembro de 2008, pelo presidente Luiz Inácio Lula da Silva. Foram criados 38 Institutos Federais espalhados pelos diversos Estados brasileiros, tendo como objetivo a oferta de cursos de educação superior, básica e profissional.

Com a expansão da Rede Federal de Educação Profissional, Científica e Tecnológica, ampliou-se consideravelmente o número de alunos matriculados e, consequentemente, a diversidade de estudantes no interior desses espaços de formação. Para atender a legislação vigente acerca da inclusão, os Institutos Federais criaram núcleos com o objetivo de promover ações relacionadas ao ingresso, à permanência e êxito dos estudantes público-alvo da Educação Especial, através da construção de uma cultura para convivência, do respeito à diversidade e da eliminação de barreiras estruturais, educacionais e atitudinais para a inclusão social e educacional dos estudantes (IFSP, 2021).

Cada Instituto Federal possui sua própria Resolução que aprova o Regulamento do NAPNE - Núcleo de Apoio às Pessoas com Necessidades Educacionais Específicas. Nesse contexto, surgiu o interesse em identificar as diferenças e semelhanças presentes nas Resoluções que aprovam os Regulamentos dos NAPNEs dos Institutos Federais de Educação, Ciência e Tecnologia, com intuito de: conhecer e analisar essas Resoluções; contribuir com o processo de reflexão acerca das Resoluções que aprovam os Regulamentos dos NAPNEs; delinear o perfil dos NAPNEs quanto à sua nomenclatura e sigla, objetivo/finalidade, público-alvo, competências, composição e financiamento.

Acredita-se que o presente estudo tenha relevância tanto para a área acadêmica quanto para o contexto de trabalho junto ao NAPNE, trazendo benefícios, como: melhora na gestão dos recursos públicos; formulação de intervenções mais adequadas à implementação das Resoluções que aprovam os Regulamentos dos NAPNEs; tomada de decisões mais efetivas, que atendam às demandas locais; maior precisão nas respostas dadas aos controles internos e externos, no que diz respeito aos diversos questionamentos quanto à efetividade das ações do NAPNE.

Durante o processo de pesquisa, foram analisadas as Resoluções que regulamentam a criação dos NAPNEs em um Instituto Federal de Educação, Ciência e Tecnologia por região do Brasil: Norte, Nordeste, Centro-Oeste, Sudeste e Sul, sendo realizada uma pesquisa 
exploratória, com abordagem qualitativa. O método escolhido para o desenvolvimento do estudo foi a pesquisa documental.

A seguir, serão apresentados: a fundamentação teórica do estudo, a metodologia utilizada para a pesquisa, os resultados alcançados e as considerações finais.

\section{FUNDAMENTAÇÃO TEÓRICA}

No Brasil, historicamente, a oferta e o acesso à educação, com destaque à educação profissional, não se deram de maneira similar para todos os cidadãos brasileiros, principalmente no que se refere à pessoa com deficiência.

Segundo Silva (2020, p.1), “o quadro educacional dessa parcela de nossa população é caracterizado, até os dias atuais, pela exclusão escolar ou pelo acesso restrito em espaços segregados, notadamente quando nos referimos à educação profissional”.

Em 1948, a Declaração Universal dos Direitos Humanos, aprovada na Assembleia Geral das Nações Unidas (ONU), significou um marco internacional no que diz respeito à legitimação da educação, incluindo a educação profissional, como direito de todos:

Todo ser humano tem o direito à instrução. A instrução será gratuita, pelo menos nos graus elementares e fundamentais. A instrução elementar será obrigatória. A instrução técnico-profissional será acessível a todos, bem como a instrução superior, esta baseada no mérito (ONU, 1948, p. 14).

No Brasil, temos a Constituição da República Federativa, de 1988, como marco políticolegal de maior expressão, onde, em seu artigo $6^{\circ}$, reconhece a educação e o trabalho como direitos sociais: "são direitos sociais a educação, a saúde, a alimentação, o trabalho, a moradia, $\mathrm{o}$ transporte, o lazer, a segurança, a previdência social, a proteção à maternidade e à infância, a assistência aos desamparados, na forma desta Constituição” (BRASIL, 1988, p. 18).

Em seu artigo 208, inciso III, a Constituição apresenta como um dos deveres do Estado com a educação o "atendimento educacional especializado aos portadores de deficiência, preferencialmente na rede regular de ensino" (BRASIL, 1988, p. 124) e em seu artigo 227, prevê como dever da família, da sociedade e do Estado assegurar à criança, ao adolescente e ao jovem o direito à profissionalização, obedecendo à preceitos, sendo um deles apresentado em seu inciso II:

(...) criação de programas de prevenção e atendimento especializado para as pessoas portadoras de deficiência física, sensorial ou mental, bem como de integração social do adolescente e do jovem portador de deficiência, mediante o treinamento para o 
trabalho e a convivência, e a facilitação do acesso aos bens e serviços coletivos, com a eliminação de obstáculos arquitetônicos e de todas as formas de discriminação (BRASIL, 1988, p. 132)

A partir da Constituição Federal de 1988, muitos outros dispositivos legais foram sancionados, proporcionando uma significativa mudança na política educacional brasileira, principalmente no tocante à educação inclusiva (SILVEIRA; NAKAMOTO, 2018). Como exemplo, podemos citar a Lei n ${ }^{\circ} 9.394$ que estabelece as diretrizes e bases da educação nacional, promulgada em 20 de dezembro de 1996:

O dever do Estado com educação escolar pública será efetivado mediante a garantia
de: (...) III - atendimento educacional especializado gratuito aos educandos com
deficiência, transtornos globais do desenvolvimento e altas habilidades ou
superdotação, transversal a todos os níveis, etapas e modalidades, preferencialmente
na rede regular de ensino (...) (BRASIL, 1996, art. $4^{\circ}$ ).

E podemos citar, também, a Lei no 13.146 de 06 de julho de 2015, que institui a Lei Brasileira de Inclusão da Pessoa com Deficiência (Estatuto da Pessoa com Deficiência): “incumbe ao poder público assegurar, criar, desenvolver, implementar, incentivar, acompanhar e avaliar: I - sistema educacional inclusivo em todos os níveis e modalidades, bem como o aprendizado ao longo de toda a vida (...)” (BRASIL, 2015, art. 28).

Em meio a esse processo de quebra de paradigma, iniciado no Brasil com a Constituição Federal de 1988, surge em meados do ano 2000, a primeira experiência de educação inclusiva da Rede Federal de Educação Profissional, Científica e Tecnológica, o Programa TEC NEP Educação, Tecnologia e Profissionalização para Pessoas com Necessidades Específicas, proposto pela Secretaria de Educação Especial e Secretaria de Educação Média e Tecnológica do Ministério da Educação - MEC (NASCIMENTO; FARIA, 2013). Conforme Silva (2017), dentro desse contexto, possivelmente foram muitas as dificuldades enfrentadas pelo Programa TEC NEP para a implementação de uma nova política, política esta,

(..) que trouxe uma outra concepção de educação em uma Rede em crescente expansão, que apresenta-se permeada por um cenário tão diversificado e complexo composto por diferentes instituições, espalhadas em todo o território brasileiro, que por si só se revelam com diferentes condições sociais, políticas e econômicas (SILVA, 2017, p. 79).

O Programa TEC NEP teve “(...) como propósito iniciar o processo de transformação da realidade atual. Além de ser uma questão de efetivação de Direitos Humanos das pessoas com necessidades especiais à Educação profissional e ao trabalho (...)” (BRASIL, 2001, p. 7). Conforme Nascimento e Faria (2013), o Programa TEC NEP foi estruturado nos seguintes momentos: Momento 1 (2000 a 2003) - Mobilização e Sensibilização; Momento 2 (2003 a 2006) 
- Consolidação dos Grupos Gestores, dos Núcleos de Atendimento às Pessoas com Necessidades Específicas - NAPNEs e a estratégia de implantação da Ação TEC NEP; Momento 3 (2007 a 2009) - Formação de Recursos Humanos, uso e desenvolvimento de tecnologia assistiva. Para Gonçalves e Azeredo (2016, p.10):

a estruturação dos Núcleos de Apoio às Pessoas com Necessidades Educacionais Especiais - NAPNEs é um dos pontos importantes deste Programa, pois as demais ações de origem interna nas instituições da Rede de Educação Profissional e Tecnológica são criadas e desenvolvidas no NAPNE.

O NAPNE, no âmbito interno das instituições federais, se constituiu como "o principal lócus de atuação do processo de inclusão" (NASCIMENTO; FARIA, 2013, p. 19), buscando estratégias para o ingresso, a permanência e o êxito escolar dos estudantes com necessidades específicas, cumprindo um "papel importante no repensar didático pedagógico e organizacional na proposta da inclusão" (PEREIRA; MONTEIRO, 2020, p. 2).

Apesar dos NAPNEs dos Institutos Federais de Educação, Ciência e Tecnologia terem sido criados a partir de uma mesma demanda do TEC NEP, não há uma resolução única que defina o funcionamento de todos os NAPNEs. A princípio, essa singularidade é uma característica positiva, pois, cada região do país possui suas próprias características e necessidades. No entanto, seria importante identificar essas diferenças, a fim de se contribuir com um debate maior no que diz respeito à construção e implementação de uma diretriz nacional.

\section{METODOLOGIA}

Para o estudo proposto, foi realizada uma pesquisa exploratória com uma abordagem qualitativa. O método escolhido para o desenvolvimento da pesquisa foi a pesquisa documental.

As fontes utilizadas na pesquisa foram as Resoluções que aprovam os Regulamentos dos NAPNEs de um Instituto Federal de Educação, Ciência e Tecnologia por região do Brasil: Instituto Federal de Educação, Ciência e Tecnologia do Amazonas - IFAM (Região Norte) Resolução no 45 - CONSUP/IFAM, de 13 de julho de 2015; Instituto Federal de Educação, Ciência e Tecnologia do Ceará - IFCE (Região Nordeste) - Resolução no 050, de 14 de dezembro de 2015; Instituto Federal de Educação, Ciência e Tecnologia de Mato Grosso do Sul - IFMS (Região Centro-Oeste) - Resolução no 026/2016, de 15 de abril de 2016; Instituto Federal de Educação, Ciência e Tecnologia de São Paulo - IFSP (Região Sudeste) - Resolução n 137 , de 04 de novembro de 2014 e Instituto Federal de Educação, Ciência e Tecnologia Catarinense - IFC (Região Sul) - Resolução no 33/2019 - CONSUPER, de 01 de julho de 2019. 
Os Institutos Federais foram escolhidos conforme a disponibilidade das Resoluções em site oficial.

Após a análise das informações obtidas por meio dos documentos, foi possível delinear o perfil dos NAPNEs quanto à sua nomenclatura e sigla, objetivo/finalidade, público-alvo, competências, composição e financiamento, identificando-se, assim, diferenças e semelhanças, bem como, limites e possibilidades.

\section{RESULTADOS}

\section{Nomenclatura e Sigla}

No que diz respeito à nomenclatura e sigla dos NAPNEs, foi percebido que nas cinco Resoluções analisadas há semelhança na sigla (todas utilizam a sigla NAPNE), mas existe, entre todas, diferença na nomenclatura do Núcleo. Observa-se que a diferença na nomenclatura dos NAPNEs se deve, primeiramente, às expressões "Núcleo de Atendimento", "Núcleo de Acessibilidade" e "Núcleo de Apoio".

Ao consultarmos o Dicio - Dicionário Online de Português (2020), vemos que a palavra atendimento (ato ou efeito de atender) está relacionada ao modo habitual através do qual são atendidas as pessoas que procuram determinado serviço; está relacionada ao local que se destina ao recebimento do público e às atividades que se referem ao contato direto com os atendidos. Já a palavra acessibilidade, está relacionada àquilo que é acessível, que oferece acesso e facilidade na aquisição do que se precisa. E a palavra apoio se refere àquilo que sustenta, que ampara, que auxilia. Nota-se que as três formas de nomenclaturas são distintas, mas ao mesmo tempo se inter-relacionam no compromisso com os direitos humanos e com a cidadania, na efetivação da inclusão social, na acolhida das demandas, na atenção e no cuidado com o público a que se destina.

Observa-se, ainda, que a diferença na nomenclatura dos NAPNEs também se deve às expressões "Necessidades Educacionais Especiais" e "Necessidades Educacionais Específicas". Ao consultarmos o Dicio - Dicionário Online de Português (2020), verificamos que tanto a palavra especiais como a palavra específicas se referem àquilo que é particular, exclusivo. No entanto, vale ressaltar que devemos tomar um cuidado maior ao utilizarmos a expressão "Necessidades Educacionais Especiais", pois muitas vezes as terminologias podem gerar efeitos negativos quando acabam criando rótulos que discriminam ou disseminam ideias preconceituosas ou pejorativas. A palavra especial, além de se referir a algo que é particular e 
próprio da pessoa, também acrescenta juízo de valor, como sendo algo ótimo e que dá vantagens extras (DICIO, 2020), podendo ser interpretada como uma situação que não necessite, portanto, de ações que a atenda.

\section{Objetivo/Finalidade}

Conforme estudo das Resoluções, os NAPNEs apresentam mais diferenças do que semelhanças no que diz respeito aos seus objetivos/finalidades.

As principais semelhanças encontradas foram: superação de barreiras arquitetônicas, comunicacionais, educacionais e atitudinais (apontada em quatro Resoluções: IFCE, IFMS, IFSP e IFC); criar e promover uma cultura para a convivência (indicada em três Resoluções: IFAM, IFMS e IFSP); promover condições para permanência dos estudantes (mencionada em duas Resoluções: IFAM e IFCE); programar e difundir as ações de inclusão (citada em duas Resoluções: IFAM e IFSP); integrar os diversos segmentos que compõem a comunidade interna e externa do Campus, desenvolvendo sentimento de corresponsabilidade na construção da ação educativa de inclusão (sinalizada em duas Resoluções: IFAM e IFSP); promover condições necessárias para o ingresso dos estudantes (presente em duas Resoluções: IFAM e IFCE).

Quando se analisou as diferenças, alguns tópicos se destacaram. São eles:

- Apenas uma Resolução (IFAM) cita o objetivo/finalidade de coordenar as ações de inclusão. Sabemos o quanto é importante a garantia, nos documentos institucionais, da definição clara dos papéis, principalmente frente à uma atuação tão específica como a do NAPNE dentro da unidade escolar;

- Apenas uma Resolução (IFCE) cita o objetivo/finalidade de promover condições necessárias para o êxito educacional. É imprescindível a criação de competências institucionais para o desenvolvimento e a aplicação de metodologias, instrumentais e currículos que materializem não só o acesso e a permanência do estudante, mas, principalmente, o seu êxito educacional e o seu ingresso no mundo do trabalho;

- Apenas uma Resolução (IFC) cita o objetivo/finalidade de fomentar estudos das questões relativas à inclusão. A pesquisa é fundamental para a construção do conhecimento e para a mobilização social. A pesquisa se faz como “(...) condição de consciência crítica e cabe como componente necessário de toda proposta emancipatória” (DEMO, 2002, p. 40). 
Sabe-se que os objetivos de um documento institucional são os norteadores de todas as ações direcionadas por eles, por isso, a importância de conhecê-los e de promovermos discussões para identificarmos se estão sendo suficientes para subsidiar todas as ações necessárias para a promoção da inclusão dos alunos, tanto na época em que foram publicados, como com o passar do tempo.

\section{Público-alvo}

Com relação ao público-alvo dos NAPNEs, constatou-se que todas as Resoluções analisadas apresentaram pessoas com deficiência como público-alvo do NAPNE, mas houve diferença em relação ao restante do público atendido. Conforme análise das cinco Resoluções, pôde-se fazer as seguintes observações:

- Quatro Resoluções (IFAM, IFCE, IFMS e IFSP) apresentaram pessoas com altas habilidades/superdotação como público-alvo do NAPNE;

- Três Resoluções (IFAM, IFCE e IFMS) apresentaram pessoas com transtornos globais do desenvolvimento como público-alvo do NAPNE, sendo que a Resolução do IFSP apresentou pessoas com transtorno do espectro autista como público-alvo do NAPNE. É importante salientar que a Classificação Estatística Internacional de Doenças e Problemas Relacionados com a Saúde (CID-10) classificava o autismo como mencionado nas três primeiras resoluções, mas que a partir de 2018, o CID-11 atualizou para a nomenclatura mencionada pelo IFSP;

- Uma Resolução (IFAM) apresentou pessoas com transtornos funcionais específicos como público-alvo do NAPNE. A Resolução caracteriza os transtornos funcionais específicos como dislexia, disortografia, disgrafia, discalculia, transtorno de atenção, hiperatividade, entre outros;

- Uma Resolução (IFMS) apresentou pessoas com transtornos de aprendizagem como público-alvo do NAPNE;

- Uma Resolução (IFC) apresentou pessoas com necessidades específicas como públicoalvo do NAPNE. 
Quanto ao público-alvo dos NAPNEs, percebe-se, no tocante às Resoluções analisadas, que a maioria das Resoluções define o atendimento ao público-alvo da educação especial, exceto o IFAM que abrange o atendimento aos alunos com transtornos funcionais específicos.

\section{Competências}

No que diz respeito às competências dos NAPNEs, após análise das Resoluções, verificou-se a predominância das semelhanças em relação às diferenças.

As semelhanças entre as competências dos NAPNEs estão relacionadas à sua atribuição de: estabelecer parcerias entre os diversos campus, com a rede pública de serviços, com fóruns, organizações, associações, representações da sociedade civil, movimentos sociais, com empresas privadas, instituições especializadas na produção de tecnologias assistivas, com a rede de educação pública e privada, com a comunidade em geral (IFAM, IFCE, IFMS, IFSP e IFC); demandar a aquisição de equipamentos, softwares e materiais didático-pedagógicos adaptados, bem como, estimular a pesquisa e o desenvolvimento de Tecnologia Assistiva (IFAM, IFCE, IFMS, IFSP e IFC); promover a superação e a remoção de barreiras arquitetônicas, atitudinais, comunicacionais e educacionais nos campus (IFCE, IFMS, IFSP e IFC); assessorar as Diretorias/dirigentes dos campus no que diz respeito à elaboração e implementação de políticas e projetos de inclusão (IFAM, IFCE, IFMS e IFC); participar do planejamento das atividades didático-pedagógicas dos campus (IFAM, IFMS, IFSP e IFC); propor políticas de inclusão junto às áreas de ensino, pesquisa, extensão e gestão dos campus (IFAM, IFCE e IFMS); realizar capacitações, formações continuadas e eventos de sensibilização para a comunidade interna e externa dos campus (IFMS, IFSP e IFC); orientar os docentes quanto às atividades didáticometodológicas, elaboração dos instrumentos de avaliação e processo de inclusão dos estudantes (IFAM, IFSP e IFC); propor projetos e a busca de recursos para execução dos mesmos (IFAM e IFMS); implementar políticas de acesso, permanência e êxito dos estudantes (IFCE e IFC); avaliar diretrizes e metas (IFAM e IFMS); oferecer suporte às políticas e projetos de inclusão nos campus (IFAM e IFMS); assessorar os demais setores dos campus (IFAM e IFC).

As diferenças encontradas entre as competências dos NAPNEs, com maior relevância e que fomentaram alguns questionamentos, foram:

- Indicar obras para o acervo das bibliotecas sobre a temática da inclusão. Competência elencada apenas em uma Resolução (IFC) e que se faz importante para disseminação do tema junto à comunidade interna e externa dos campus; 
- Monitorar políticas e projetos de inclusão, bem como a busca de recursos para a execução deles. Competência elencada em apenas uma Resolução (IFAM). É fato que o monitoramento é uma ferramenta imprescindível para o bom gerenciamento de uma política pública. O monitoramento é a base para a realização de uma avaliação satisfatória, mas cai no esquecimento frente às demandas e tarefas do dia a dia;

- Desenvolver ações de cunho multiprofissional e multidisciplinar. Competência elencada em apenas uma Resolução (IFAM) e tão necessária para a execução do trabalho do NAPNE, trabalho este, que exige a atuação de uma equipe composta por profissionais que ao mesmo tempo que possuem diferentes especialidades e diferentes saberes, se completam no atendimento das demandas.

\section{Composição}

No que se refere ao número mínimo de integrantes da equipe, apenas três Resoluções apontaram esse item (IFAM, IFCE e IFSP). Na Resolução do IFAM, foi apontado um número mínimo de dois integrantes, sendo eles, obrigatoriamente, servidores efetivos do Instituto. Já na Resolução do IFCE, foi indicado um número mínimo de três integrantes. Na Resolução do IFSP, deve fazer parte da equipe, necessariamente, um pedagogo, um assistente social, um psicólogo e um técnico em assuntos educacionais, todos lotados na Coordenadoria Sociopedagógica da Instituição.

A presença de Coordenador na composição do NAPNE foi encontrada em três Resoluções (IFAM, IFMS e IFC). A Resolução do IFSP aponta a presença de um Representante eleito pela maioria dos membros do NAPNE.

Apenas uma Resolução (IFC) aponta a figura de um Vice Coordenador do NAPNE e três Resoluções (IFAM, IFSP e IFC) apontam a figura de um Secretário.

A presença de uma equipe multi/interdisciplinar na composição do NAPNE foi indicada em três Resoluções (IFCE, IFMS e IFSP) e a possibilidade de participação de membros da comunidade escolar foram indicadas em duas Resoluções (IFMS e IFSP).

As Resoluções do IFCE e do IFC citam como componentes do NAPNE os discentes, sendo que a Resolução do IFCE prevê que dentre os membros titulares do NAPNE deverá haver pelo menos um discente regularmente matriculado e que seja dada prioridade aos discentes que possuam alguma deficiência. As mesmas Resoluções apontam a possibilidade de 
inclusão, como membros do NAPNE, de pessoas da comunidade externa/membros da sociedade civil, sendo que a Resolução do IFC determina que sejam pessoas com atuação em movimentos sociais e que tenham afinidade com a temática.

A Resolução do IFAM, menciona a possibilidade de o NAPNE agregar membros colaboradores junto à sua composição.

No que diz respeito à indicação dos membros que compõem o NAPNE, observou-se que: na Resolução do IFAM, apenas o Coordenador é indicado pelo Diretor Geral do campus; as Resoluções do IFCE e IFSP preveem que a composição da equipe do NAPNE dar-se-á por meio de adesão voluntária; na Resolução do IFMS, o coordenador é indicado pelo Diretor Geral do campus, a equipe interdisciplinar é designada, também, pelo Diretor Geral do campus e os demais membros se candidatam voluntariamente; a Resolução do IFC não cita a forma de adesão dos membros que compõem o NAPNE.

Quanto às exigências para participação no NAPNE, foi possível constatar: na Resolução do IFAM, encontramos a indicação pela preferência de pessoas com cursos nas áreas de educação especial e educação inclusiva e que tenham interesse em desenvolver ações dentro desta temática; a Resolução do IFCE aponta a preferência por pessoas que possuam conhecimento e/ou experiência na atuação em políticas voltadas às pessoas com deficiência e/ou ações afirmativas; na Resolução do IFMS, os indicados ao NAPNE deverão, preferencialmente, ter formação e/ou experiência em educação especial e inclusiva ou áreas afins e na falta de pessoas com esses requisitos, justifica-se a indicação de outro, desde que o mesmo se comprometa a buscar o conhecimento exigido; a Resolução do IFSP menciona que, além dos profissionais específicos, os demais membros do NAPNE devam se identificar com o tema da inclusão e estar dispostos a integrarem-se aos projetos e a desenvolverem as ações da educação inclusiva no campus; a Resolução do IFC não apresentou exigências quanto à participação no NAPNE.

É importante ressaltar que, em todas as Resoluções analisadas, não foi citada a presença do professor de Atendimento Educacional Especializado - AEE na composição do NAPNE, profissional especializado que, em parceria com os professores responsáveis pelas turmas, identifica as barreiras arquitetônicas, atitudinais, comunicacionais e educacionais que impedem a efetivação do processo de ensino-aprendizagem dos estudantes com necessidades educacionais específicas e escolhe as formas de trabalho adequadas para cada estudante. A 
ausência desse profissional nas Resoluções analisadas, reflete a necessidade da incorporação desse profissional ao quadro de servidores dos Institutos Federais em questão, tendo em vista a promoção de uma educação inclusiva, que garante o direito de todos à educação, pressupondo igualdade de oportunidade e o reconhecimento das diferenças humanas.

\section{Financiamento}

Analisando as Resoluções, primeiramente, podemos observar que apenas três Resoluções apresentam informações relativas ao financiamento do NAPNE (IFAM, IFCE e IFMS), demonstrando uma fragilidade quanto à abordagem desse tema junto à formulação dos Regulamentos dos Núcleos.

Podemos constatar, também, que há diferenças nas abordagens identificadas nas três Resoluções. Na Resolução do IFAM, o planejamento orçamentário anual do NAPNE limita-se à previsão de recursos para a realização de encontros institucionais. Na Resolução do IFCE há uma abordagem mais aprofundada, onde temos a previsão da inclusão das ações do NAPNE em documentos oficiais do campus e temos a previsão da solicitação anual do orçamento destinado à essas mesmas ações. E, por fim, na Resolução do IFMS, encontramos apenas as possíveis fontes de financiamento e manutenção do NAPNE.

A análise das Resoluções possibilitou a identificação da existência de um processo inovador que vê a questão do financiamento do NAPNE como parte importante de sua regulamentação. No entanto, foi possível perceber, também, que muito se precisa avançar no que diz respeito à garantia de um orçamento que assegure, fielmente, os recursos físicos, materiais e humanos necessários ao atendimento das demandas recebidas pelo NAPNE.

\section{CONSIDERAÇÕES FINAIS}

Propusemos neste estudo, analisar as Resoluções que regulamentam a criação dos NAPNEs em um Instituto Federal de Educação, Ciência e Tecnologia por região do Brasil, identificando suas semelhanças e diferenças quanto à sua nomenclatura e sigla, objetivo/finalidade, público-alvo, competências, composição e financiamento.

De acordo com o estudo das Resoluções, há a predominância das diferenças. Dessa forma, consideramos que se evidenciou, pela pesquisa realizada, a necessidade da elaboração de uma diretriz nacional que minimize as disparidades encontradas entre os NAPNEs, diretriz esta, 
que possa oferecer um traçado metodológico a se seguir, respeitando-se as particularidades de cada instituição e o contexto social e econômico no qual estão inseridas.

Ao delinearmos os perfis dos NAPNEs, foi possível observarmos algumas fragilidades/limitações presentes em seus Regulamentos que abrem perspectivas/possibilidades para novas pesquisas, sendo elas: a priorização do acesso e da permanência do estudante em detrimento do seu êxito educacional; a necessidade da institucionalização do financiamento do NAPNE como parte essencial de sua regulamentação e a demanda da incorporação do professor de AEE ao quadro de servidores dos Institutos Federais de Educação, Ciência e Tecnologia.

\section{REFERÊNCIAS}

BRASIL. Constituição da República Federativa do Brasil: texto constitucional promulgado em 05 de outubro de 1988, com as alterações determinadas pelas Emendas Constitucionais de Revisão no 1 a 6/94, pelas Emendas Constitucionais no 1/92 a 91/2016 e pelo Decreto Legislativo no 186/2008. Brasília: Senado Federal, Coordenação de Edições Técnicas, 2016. Disponível em:

< https://www2.senado.leg.br/bdsf/bitstream/handle/id/518231/CF88 Livro EC91 2016. pdf>. Acesso em: 24 de jun. 2020.

BRASIL. Lei no 9.394, de 20 de dezembro de 1996. Brasília, 1996. Disponível em: <http://www.planalto.gov.br/ccivil 03/leis/19394.htm>. Acesso em: 24 jul. 2020.

BRASIL. Programa TEC NEP: educação, tecnologia e profissionalização para pessoas com necessidades especiais. Brasília: Secretaria de Educação Média e Tecnológica/Secretaria de Educação Especial, 2001.

BRASIL. Lei no 13.146, de 06 de julho de 2015. Brasília, 2015. Disponível em: < http://www.planalto.gov.br/ccivil 03/ ato2015-2018/2015/lei/113146.htm >. Acesso em: 24 jul. 2020.

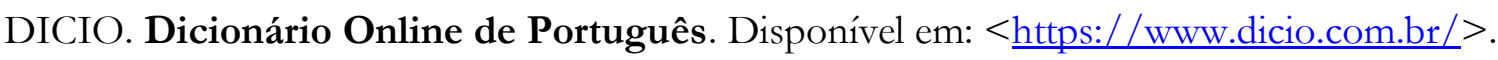
Acesso em: 13 de jun. 2020.

DEMO, P. Desafios modernos da educação. 12 ed. Petrópolis: Vozes, 2002. 
GONÇALVES, Lizandra Falcão; AZEREDO, Marta Roseli de. TECNEP interface entre educação profissional e tecnológica e educação especial. II Fórum Internacional de Educação, VI Seminário Nacional de Pesquisa em Educação, XIV Fórum Nacional de Educação, XVII Seminário Regional de educação Básica. Santa Cruz do Sul: Universidade de Santa Cruz do Sul, 2016. Disponível em:

$<$ https://online.unisc.br/acadnet/anais/index.php/sepedu/article/download/14873/3470>. Acesso em: 25 de jun. 2020.

IFAM - Instituto Federal de Educação, Ciência e Tecnologia do Amazonas. Resolução no 45 - CONSUP/IFAM, de 13 de julho de 2015. Disponível em:

$<\underline{\text { http://www2.ifam.edu.br/pro- }}$ reitorias/ensino/documentos/resolucoes/2015/arquivos/resolucao-no-45-aprova-regimentointern-do-napne.pdf/view >. Acesso em: 12 jun. 2020.

IFC - Instituto Federal de Educação, Ciência e Tecnologia Catarinense. Resolução $\mathbf{n}^{\mathbf{o}}$ 33/2019 - CONSUPER, de 01 de julho de 2019. Dispõe sobre a Política de Inclusão e Diversidade do Instituto Federal Catarinense (IFC). Blumenau, 2019. Disponível em: $<$ http://estudante.ifc.edu.br/2019/09/18/politica-de-inclusao-e-diversidade-do-institutofederal-catarinense/>. Acesso em: 12 de jun. 2020.

IFCE - Instituto Federal de Educação, Ciência e Tecnologia do Ceará. Resolução no 050 de 14 de dezembro de 2015. Aprova o Regulamento dos NAPNEs do IFCE. Disponível em: $<$ https://ifce.edu.br/proext/acessibilidade/arquivos/resolucao-no-050-2015napne.pdf/view >. Acesso em: 12 jun. 2020.

IFMS - Instituto Federal de Educação, Ciência e Tecnologia de Mato Grosso do Sul. Resolução no 026/2016, de 15 de abril de 2016. Campo Grande, 2016. Disponível em: < http://www.ifms.edu.br:8090/centrais-de-conteudo/documentosinstitucionais/resolucoes/resolucao-026-aprova-regulamento-napne-15-04-2016.pdf/view $>$. Acesso em: 12 de jun. 2020.

IFSP - Instituto Federal de Educação, Ciência e Tecnologia de São Paulo. Resolução no 137, de 04 de novembro de 2014. Aprova o Regulamento do Núcleo de Apoio às Pessoas com Necessidades Educacionais Específicas (NAPNE). São Paulo, 2014a. Disponível em: < https://ifsp.edu.br/images/reitoria/Resolucoes/resolucoes2014/Resol 137 AprovaRegulamento-NAPNE.pdf>. Acesso em: 12 de jun. 2020. 
IFSP - Instituto Federal de Educação, Ciência e Tecnologia de São Paulo. Ações Inclusivas. 2021. Disponível em: < https://www.ifsp.edu.br/component/content/article/1472>. Acesso em: 04 de fev. 2021.

NASCIMENTO, Franclin; FARIA, Rogério. “A questão da inclusão na rede federal de educação profissional, científica e tecnológica, a partir da Ação TEC NEP”. In: NASCIMENTO, Franclin Costa do; FLORINDO, Girlane Maria Ferreira; SILVA, Neide Samico da (orgs.). Educação profissional e tecnológica inclusiva: um caminho em construção. Brasília: Instituto Federal de Educação, Ciência e Tecnologia de Brasília, 2013, p. 13-23.

ONU. Declaração Universal dos Direitos Humanos (1948). Disponível em: $<$ https://nacoesunidas.org/wp-content/uploads/2018/10/DUDH.pdf $>$. Acesso em: 24 de jun. 2020.

PEREIRA, Miriam Lúcia Reis Macedo; MONTEIRO, Eneida Aparecida M. NAPNE: as contribuições e os desafios frente a inclusão. Disponível em:

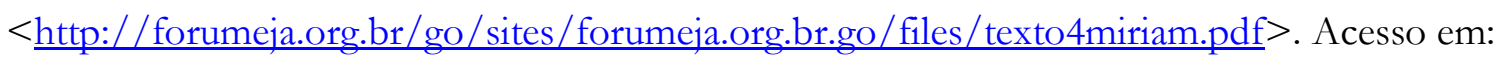
25 de jun. 2020 .

SILVA, Izaura Maria de Andrade da. Políticas de educação profissional para pessoas com deficiência. Disponível em: < http://www.anped.org.br/sites/default/files/gt15-4258int.pdf>. Acesso em: 24 de jun. 2020.

SILVA, Rosilene Lima da. O Núcleo de Atendimento às Pessoas com Necessidades Específicas (NAPNE) no Institutos Federais da Região Nordeste do Brasil: Desafios Políticos e Perspectivas Pedagógicas. 2017. 180 f. Dissertação (Mestrado em Educação, Contextos Contemporâneos e Demandas Populares) - Instituto de Educação e Instituto Multidisciplinar, Universidade Federal Rural do Rio de Janeiro, Seropédica, 2017.

SILVEIRA, Marieles da; NAKAMOTO, Paula Teixeira. A educação profissional e a educação da pessoa com deficiência. Anais do V Seminário de Pós-Graduação - V SIMPÓS, v. 5, 2018. Disponível em:

$<$ https://iftm.edu.br/simpos/2018/anais/689-020Pronto\%20ANAIS.pdf $>$. Acesso em 25 de jun. de 2020. 